\title{
Open problems in theory of nuclear open quantum systems
}

\author{
N. Michel ${ }^{1}$, W. Nazarewicz ${ }^{2-4}$, J. Okołowicz ${ }^{5}$ and M. \\ Płoszajczak $^{6}$ \\ ${ }^{1}$ Department of Physics, Post Office Box 35 (YFL), FI-40014 University of \\ Jyväskylä, Finland \\ 2 Department of Physics and Astronomy, University of Tennessee, Knoxville, \\ Tennessee 37996, USA \\ ${ }^{3}$ Physics Division, Oak Ridge National Laboratory, Oak Ridge, Tennessee 37831, \\ USA \\ ${ }^{4}$ Institute of Theoretical Physics, University of Warsaw, ul. Hoża 69, PL-00-681 \\ Warsaw, Poland \\ ${ }^{5}$ Institute of Nuclear Physics, Polish Academy of Sciences, Radzikowskiego 152, \\ PL-31342 Kraków, Poland \\ ${ }^{6}$ Grand Accélérateur National d'Ions Lourds (GANIL), CEA/DSM - CNRS/IN2P3, \\ BP 55027, F-14076 Caen Cedex, France \\ E-mail: witek@utk.edu, ploszajczak@ganil.fr
}

\begin{abstract}
Is there a connection between the branch point singularity at the particle emission threshold and the appearance of cluster states which reveal the structure of a corresponding reaction channel? Which nuclear states are most impacted by the coupling to the scattering continuum? What should be the most important steps in developing the theory that will truly unify nuclear structure and nuclear reactions? The common denominator of these questions is the continuum shell-model approach to bound and unbound nuclear states, nuclear decays, and reactions.
\end{abstract}




\section{Introduction}

The nuclear shell model was proposed almost sixty years ago [1, 2]. Soon afterwards, the interacting shell model (ISM) was developed by Lane [3], Kurath [4], and others (see [5] for a recent review). The ISM-based description of an evolution of the nucleonic coupling scheme from the $L S$ to $j j$ coupling with increasing mass number [3, 4, 6], provided foundations of modern nuclear structure theory and helped to understand and categorize a wealth of data on nuclear levels, moments, collective excitations, electromagnetic and $\beta$ decays, and various particle decays [7].

In its traditional form, ISM describes the nucleus as a closed quantum system: nucleons occupy bound, hence well localized, single-particle orbits of an infinite (e.g., harmonic oscillator) potential and are isolated from the environment of unbound scattering states that are not square integrable. Since the scattering continuum is not considered explicitly, the presence of branch points (decay thresholds) and doublepoles of the scattering matrix ( $S$-matrix) is neglected. The divide between the discrete states and the scattering continuum, i.e., the focus on one or another, has unfortunately become a kind of paradigm. In the long term, this has led to an artificial separation of nuclear structure from nuclear reactions, and hindered a deeper understanding of nuclear properties. Indeed, many structural properties of the nucleus are determined by means of nuclear collisions. Hence, the knowledge of nuclear structure depends on nuclear reactions and vice versa, and this cries out for a unified theoretical framework.

The first attempt in this direction came from Feshbach [8, 9] who expressed the collision matrix of the optical model using matrix elements of the Hamiltonian. This development gave a strong push to the ISM approach to nuclear reactions [10, 11, 12, 13] (see introduction in Ref. [14] for a detailed historical account) and led to various formulations of the continuum shell model (CSM) [15, 16, 17, 18, 19, 20]. A modern version of CSM in the Hilbert space, the Shell Model Embedded in the Continuum (SMEC) [19, 21], provides a unified description of the structure and reactions with up to two nucleons in the scattering continuum using realistic ISM Hamiltonians. Nevertheless, the fully symmetric treatment of bound and scattering states in the multiparticle wave function is still too ambitious a goal [22].

A different attempt to formulate the ISM for open quantum systems has been proposed recently [23, 24, 25, 26] within the Berggren ensemble [27]. The resulting complex-energy open quantum system extension of the ISM, the Gamow Shell Model (GSM), can be conveniently formulated in the Rigged Hilbert Space (Gel'fand triple) [28, 29], which encompasses Gamow states [30, 31], and is suitable for extending the quantum mechanics into the domain of time-asymmetric processes (e.g. decays). The GSM offers a fully symmetric treatment of bound, resonance, and scattering singleparticle states but, until now, has been primarily used in the context of nuclear structure. (For a recent review of the complex-energy shell model, see Ref. [32].)

In this paper, we shall draw attention to salient threshold effects related to generic properties of branch points and $S$-matrix poles that are expected to impact structural 
properties of nuclei. In Sec. 2.2, we shall use the phase rigidity indicator to demonstrate effects of the branch point at the particle emission threshold and the exceptional point (the double-pole of the $S$-matrix) on the configuration mixing in the continuum. Section 2.3 discusses the stability of ISM eigenfunctions in the neighborhood of the reaction threshold. Finally, in Sec. 2.4 we outline necessary developments to achieve a unified theory of structure and reactions offering a symmetric treatment of bound, resonance, and scattering states.

\section{Salient near-threshold phenomena}

At low excitation energies, well-bound nuclei can be considered as closed quantum systems, well described by the standard ISM or its modern versions such as the no-core shell model [33, 34, 35]. Moving towards drip lines, or higher in excitation energy, the continuum coupling becomes gradually more important, changing the nature of weakly bound states. (Properties of unbound states are impacted by couplings to reaction channels.) In this regime, the chemical potential has a magnitude similar to the pairing gap; hence, the system is dominated by many-nucleon correlations which no longer can be considered as small perturbations atop the average potential [36]. Many-body states in neighboring nuclear systems with different proton and neutron numbers become interconnected via continuum, forming correlated domains (clusters) of quantum states. At present, little is known about the basic features of such clusters, e.g. a typical 'cluster size' and its dependence on binding properties of participating states.

In standard network problems, like the percolation problem [37, 38], different phases of correlated domains are formed depending on the mean network activity with respect to some threshold. Analogously, one expects that the key to understanding the formation of correlated domains of quantum states is a near-threshold behavior of a single network member. In the correlation-dominated regime of a nuclear system, the percolation threshold(s) should be identified with reaction threshold(s).

What can be said about properties of many-body systems in the narrow range of energies around the reaction threshold? Are those properties independent of any particular realization of the Hamiltonian? Below, we shall try to address these questions in the framework of SMEC, but essentials of this discussion are valid in the GSM approach as well.

\subsection{The main ingredients of the continuum shell model}

To facilitate further discussion, we shall now recall fundamentals of SMEC. The Fock space of an $A$-particle system consists of the set of square-integrable functions $\mathcal{Q} \equiv\left\{\Phi_{i}^{(A)}\right\}$, internal space described by the standard ISM, and the space of embedding scattering states $\mathcal{P} \equiv\left\{\zeta_{E}^{c}\right\}$. These two orthogonal and complementary subspaces of the Fock space are obtained by the projection formalism. In one-particle continuum

problems, reaction channels ' $c$ ' correspond to the motion of an unbound nucleon in a 


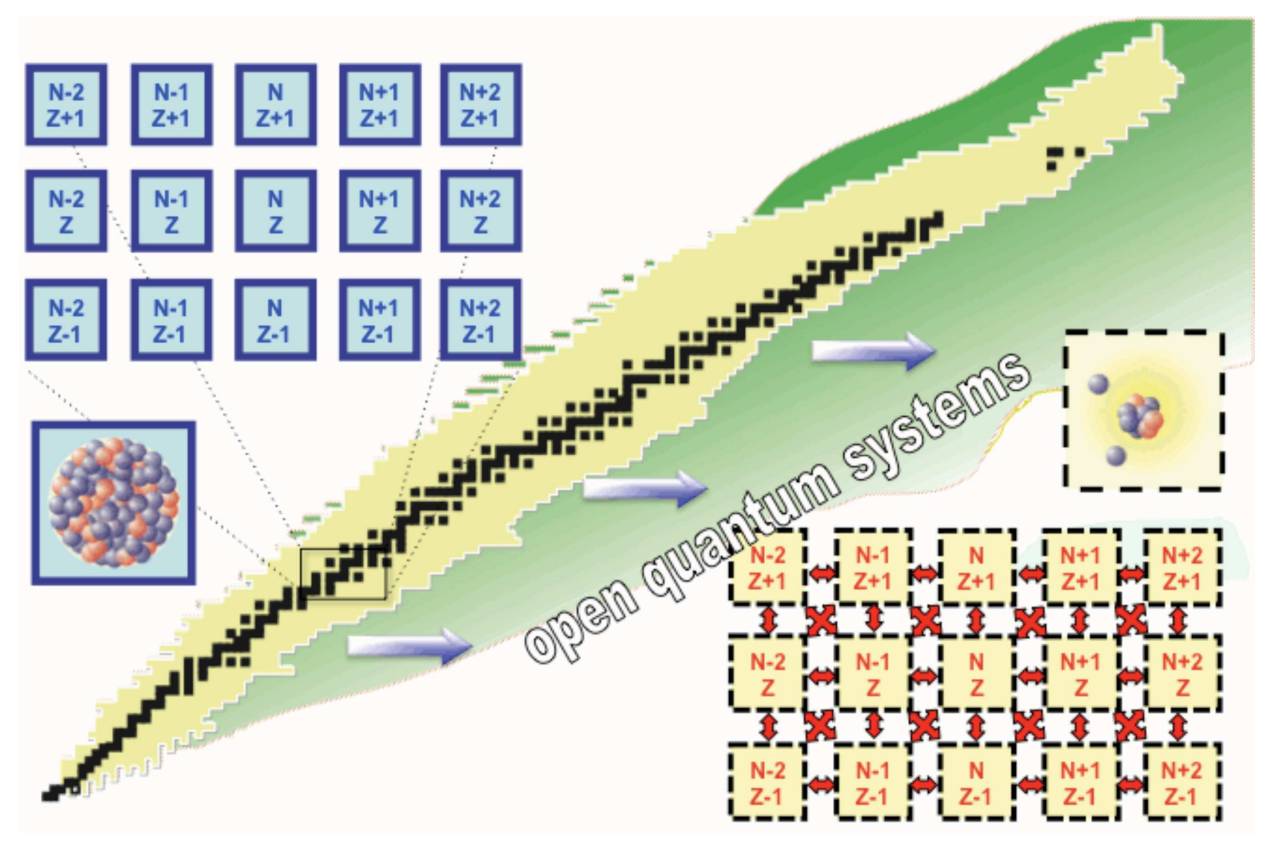

Figure 1. At low excitation energy, well-bound nuclei close to the stability valley can be described as closed quantum systems. When moving towards the particle drip lines, nuclei with different proton and neutron numbers become interconnected via continuum space, i.e., reaction channels, forming correlated domains of quantum states.

state $(l j)$ relative to the daughter nucleus in some ISM eigenstate $\Phi_{k}^{(A-1)}$. An open quantum system description of 'internal' dynamics, i.e., in $\mathcal{Q}$, includes couplings to the 'environment' of scattering states and decay channels and is given by the energydependent effective Hamiltonian:

$$
\mathcal{H}_{Q Q}(E)=H_{Q Q}+W(E),
$$

where $H_{Q Q}$ is the ISM Hamiltonian (the closed quantum system Hamiltonian) in the internal space $\mathcal{Q}$ and

$$
W(E)=H_{Q P} G_{P}^{(+)}(E) H_{P Q}
$$

is the energy-dependent term describing both virtual particle excitations and irreversible decays to the environment of reaction channels. In the latter expression, $G_{P}^{(+)}(E)$ is the Green's function for the motion of a single nucleon in the continuum, and $E$ is the nucleon's energy (i.e., the scattering energy). The external mixing of two ISM states $i$ and $j$ due to $W(E)$ consists of the Hermitian principal value integral $W^{R}(E)$ describing virtual excitations to the continuum and the anti-Hermitian residuum $W^{I}(E)=-(i / 2) \mathbf{V} \mathbf{V}^{T}$ in a dyadic form that represents decay out of the internal space $\mathcal{Q}$. The $M \times \Lambda$ matrix $\mathbf{V}=\left\{V_{i}^{c}\right\}$ denotes the amplitudes connecting the ISM state $\Phi_{i}$ $(i=1, \cdots, M)$ to the reaction channel $c(c=1, \cdots, \Lambda)$ [22].

At energies below the lowest reaction threshold, the effective Hamiltonian is Hermitian, i.e., $W^{I}=0$. Above the first threshold, the non-Hermitian part of $\mathcal{H}_{Q Q}(E)$ describes the irreversible decay from the internal space. The effective Hamiltonian in 
this case becomes complex symmetric. Each eigenstate of $\mathcal{H}_{Q Q}(E)$ is coupled to states in neighboring nuclei via a network of reaction channels, either closed or open. The contribution of different reaction channels to the total continuum coupling is highly non-uniform and spans over a considerable range of excitation energies [39]. Since the Hamiltonian (1) depends explicitly on the energy, it is highly non-linear. Moreover, the continuum-coupling term generates effective many-body interactions in the internal space, even if it is originally two-body in the full space.

Effects of the resulting many-body interactions have been studied by considering the continuum-coupling correction to the binding energy for the chains of oxygen and fluorine isotopes [39, 40]. It has been found that the induced many-body interactions explain a significant shift in the neutron drip line for fluorine isotopes as compared to the oxygens. Another explanation, explicitly invoking effective three-body interaction, has recently been suggested in Ref. [41]. These two scenarios could be difficult to distinguish.

Eigenstates of the open quantum system Hamiltonian $\mathcal{H}_{Q Q}(E)$ are biorthogonal. The left $\left|\Psi_{j}\right\rangle$ and right $\left|\Psi_{j}\right\rangle$ eigenstates have complex conjugate wave functions. The Hermitian conjugation of $\mathcal{H}_{Q Q}$ switches the left and right vectors. The orthonormality condition in the biorthogonal basis can be written as $\left\langle\Psi_{\tilde{j}} \mid \Psi_{k}\right\rangle=\delta_{j k}$.

The diagonalization of the effective SMEC Hamiltonian can be achieved by means of an orthogonal transformation. The resulting eigenvalues are real if all reaction channels are closed. Above the threshold, the transformation becomes non-unitary:

$$
\Phi_{i} \longrightarrow \Psi_{j}=\sum_{i} b_{j i} \Phi_{i}
$$

and it yields complex eigenvalues. Physical resonances can be identified with narrow poles of the $S$-matrix [42, 27, 32], or using the Breit-Wigner approach, which leads to a fixed-point condition [20, 22, 43].

2.2. How to monitor effects of branch points and avoided level crossings in the complex energy plane?

The energy-dependent term (2) describing the coupling of closed quantum system eigenfunctions $\Phi_{i}$ to the environment of scattering states does not act uniformly on all ISM states. A useful indicator of effects caused by an anti-Hermitian coupling term $W^{I}(E)$ on biorthogonal eigenfunctions $\left\{\Psi_{k}\right\}$ of the effective Hamiltonian is the phase rigidity [44, 45, 46]:

$$
r_{j}=\frac{\left\langle\Psi_{\tilde{j}} \mid \Psi_{j}\right\rangle}{\left\langle\Psi_{j} \mid \Psi_{j}\right\rangle}
$$

given by the ratio of biorthogonal and Hermitian norms of an eigenfunction. The indicator (4) can also be written in a form [46]:

$$
r_{j}=e^{2 i \theta_{j}} \frac{\int d r\left(\left|\operatorname{Re} \bar{\Psi}_{j}(r)\right|^{2}-\left|\operatorname{Im} \bar{\Psi}_{j}(r)\right|^{2}\right)}{\int d r\left(\left|\operatorname{Re} \bar{\Psi}_{j}(r)\right|^{2}+\left|\operatorname{Im} \bar{\Psi}_{j}(r)\right|^{2}\right)},
$$


which better illustrates its physical meaning. In this expression, the angle $\theta_{j}$ arises from a transformation of $\Psi_{j}$ so that $\operatorname{Re} \bar{\Psi}_{j}$ and $\operatorname{Im} \bar{\Psi}_{j}$ are orthogonal and it characterizes the degree to which the eigenfunction $\Psi_{j}$ is complex.

Phase rigidity varies between 1 and 0 . It equals 1 for bound state eigenfunctions. For unbound states, the condition $r_{j}=1$ means that the non-Hermitian coupling $W^{I}(E)$ exerts a negligible effect on the structure of an eigenfunction, i.e., its biorthogonal and Hermitian norms are identical. The energy variation of $r_{j}$ is an internal property of a considered open quantum system and measures a mutual influence of the neighboring resonances.

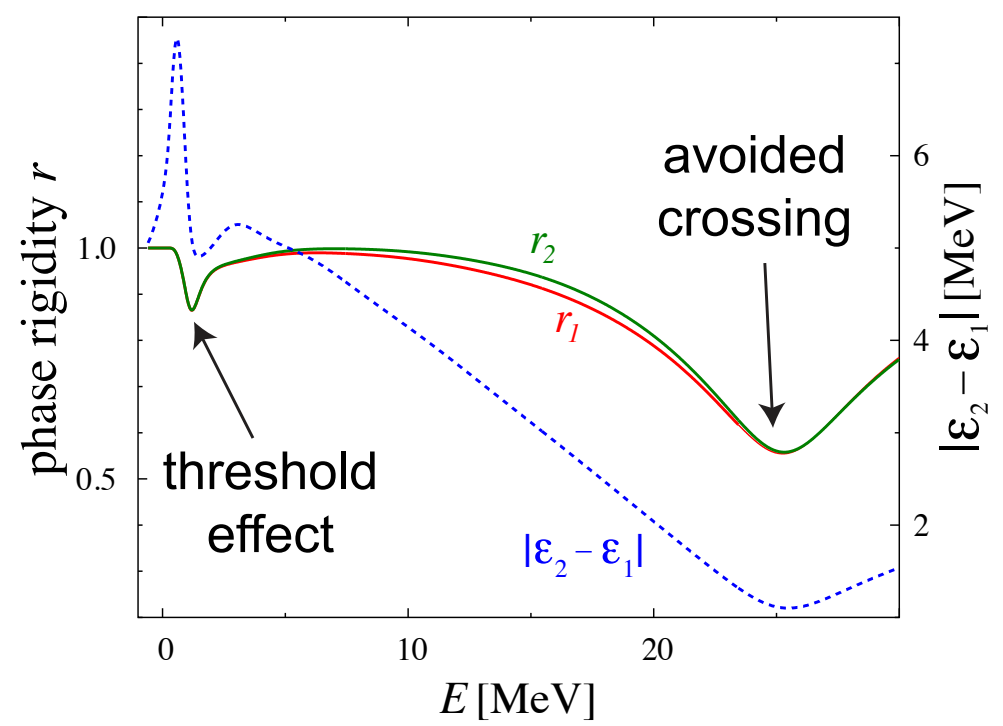

Figure 2. The energy dependence of the phase rigidity for the wave functions of the $0_{1}^{+}$and $0_{2}^{+}$SMEC states of ${ }^{16} \mathrm{Ne}$. The dashed curve shows the difference between energies of these states in the complex energy plane.

Figure 2 shows the phase rigidity as a function of the scattering energy $E$ for the two lowest-energy $0^{+}$levels of the effective Hamiltonian of ${ }^{16} \mathrm{Ne}$. These SMEC calculations are performed in a $p_{1 / 2}, d_{5 / 2}, s_{1 / 2}$ model space. For $H_{Q Q}$ we use the ZBM Hamiltonian [47]. The residual coupling $H_{Q P}$ between the internal space and the surrounding continuum is generated by the contact force: $H_{Q P}=H_{P Q}=V_{0} \delta\left(r_{1}-r_{2}\right)$ with $V_{0}=-1100 \mathrm{MeV} \mathrm{fm}^{3}$. The $0^{+}$ISM eigenstates are coupled through the common physical one-proton emission channels $\left[{ }^{15} \mathrm{~F}\left(I^{\pi}\right) \otimes{ }^{1} \mathrm{H}(l j)\right]_{E^{\prime}}^{J^{\pi}}$ with $I^{\pi}=1 / 2^{+}, 5 / 2^{+}$, and $1 / 2^{-}$which have thresholds at $E=0$ (elastic channel), $0.67 \mathrm{MeV}$, and $2.26 \mathrm{MeV}$, respectively. (Each physical channel, specified by a states $I^{\pi}$ and $J^{\pi}$ in daughter ${ }^{A-1} X$ and parent ${ }^{A} Y$ nuclei, respectively, may correspond to a number of different mathematical channels characterized by different $(l j)$-values of a nucleon which are allowed by the coupling of $I^{\pi}$ and $J^{\pi}$.) These are all possible one-proton emission channels in ${ }^{16} \mathrm{Ne}$, described in ZBM space.

One can notice two local variations of the phase rigidity for $0_{1}^{+}$and $0_{2}^{+}$ 
eigenfunctions. The first one appears close to the elastic channel threshold whereas the second one, at higher energies, indicates the avoided level crossing between $0_{1}^{+}$ and $\mathrm{O}_{2}^{+}$eigenvalues in the complex energy plane. Avoided level crossings are traces of exceptional points [48, 49, 50] in the parameter space of the effective Hamiltonian [51, 52]. The dashed curve in Fig. 2 shows a distance in the complex energy plane between the two $0^{+}$eigenvalues. The minimum in $\Delta \varepsilon=\left|\varepsilon_{1}-\varepsilon_{2}\right|(E)$ coincides with the minimum in $r(E)$ at a position of the avoided crossing. However, neither maximum nor minimum of $\Delta \varepsilon$ coincides with the minimum of the phase rigidity around the elastic reaction threshold.

A striking example of the configuration mixing caused by the coupling term $W(E)$ is shown in Fig. 3. The phase rigidity for $2_{i}^{+}(i=1, \cdots, 4)$ states in ${ }^{16} \mathrm{Ne}$ is plotted here for $V_{0}=-1357 \mathrm{MeV} \mathrm{fm}{ }^{3}$. The $2^{+}$states are interconnected via the coupling to the inelastic channel: $\left[{ }^{15} \mathrm{~F}\left(5 / 2^{\pi}\right) \otimes{ }^{1} \mathrm{H}(l j)\right]_{E^{\prime}}^{2^{+}}$. One can clearly see that at this value of the continuum coupling strength there appears the degeneracy (exceptional point) of $2_{3}^{+}$and $2_{4}^{+}$levels at which the phase rigidity drops abruptly to 0 . The remaining $2^{+}$ eigenfunctions are mere spectators of this violent wave function rearrangement. At low

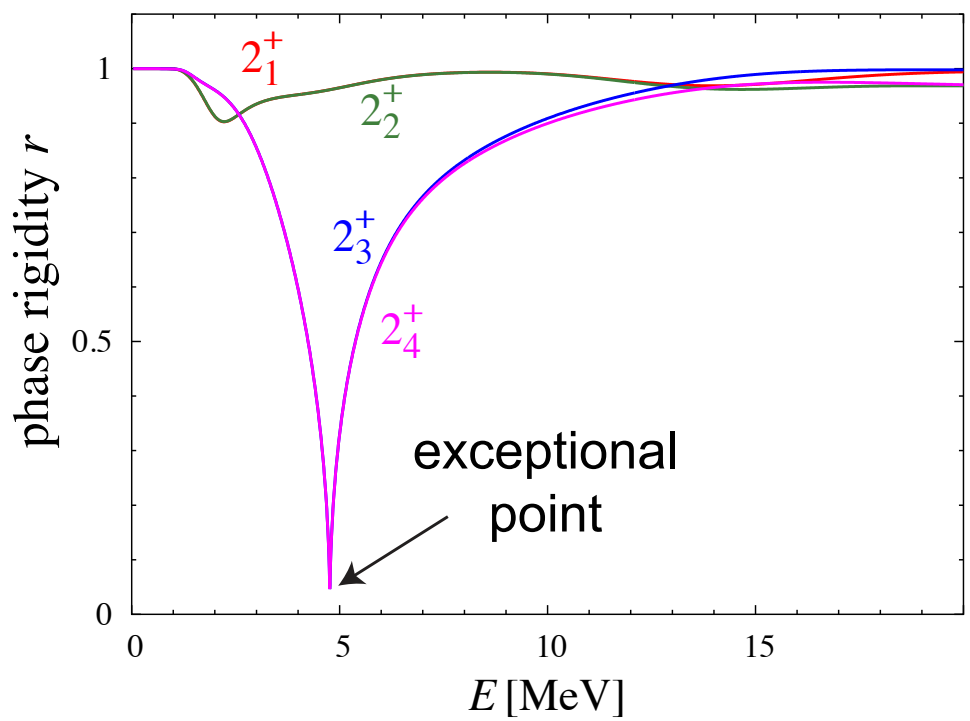

Figure 3. The energy dependence of the phase rigidity for lowest $2_{i}^{+}(i=1, \cdots, 4)$ eigenfunctions of the SMEC Hamiltonian in ${ }^{16} \mathrm{Ne}$. The $2_{3}^{+}$and $2_{4}^{+}$eigenvalues form an exceptional point at $E \simeq 4.8 \mathrm{MeV}$.

energies, close to the branch point at the channel threshold $(E=0.67 \mathrm{MeV})$, one can see a strong mixing of $2_{1}^{+}$and $2_{2}^{+}$eigenfunctions.

\subsection{Near-threshold instability of the wave function}

Both the branch points associated with reaction thresholds and the avoided level crossings are essential elements of the configuration mixing in open quantum systems. Abrupt variations of the phase rigidity as a function of the energy for certain eigenstates 
of the effective Hamiltonian are indicative of wave function instability. Another indicator of such instabilities could be the continuum-coupling energy correction, $E_{\text {corr }}(E)=$ $\left\langle\Phi_{i}|W(E)| \Phi_{i}\right\rangle$, to the ISM eigenvalue $\left\langle\Phi_{i}\left|H_{Q Q}\right| \Phi_{i}\right\rangle$. Figure 4 (left) shows the continuumcoupling correction for the ground states of oxygen isotopes calculated for a single channel corresponding to a neutron coupled to the ground state in the daughter nucleus ${ }^{A-1} \mathrm{O}$.

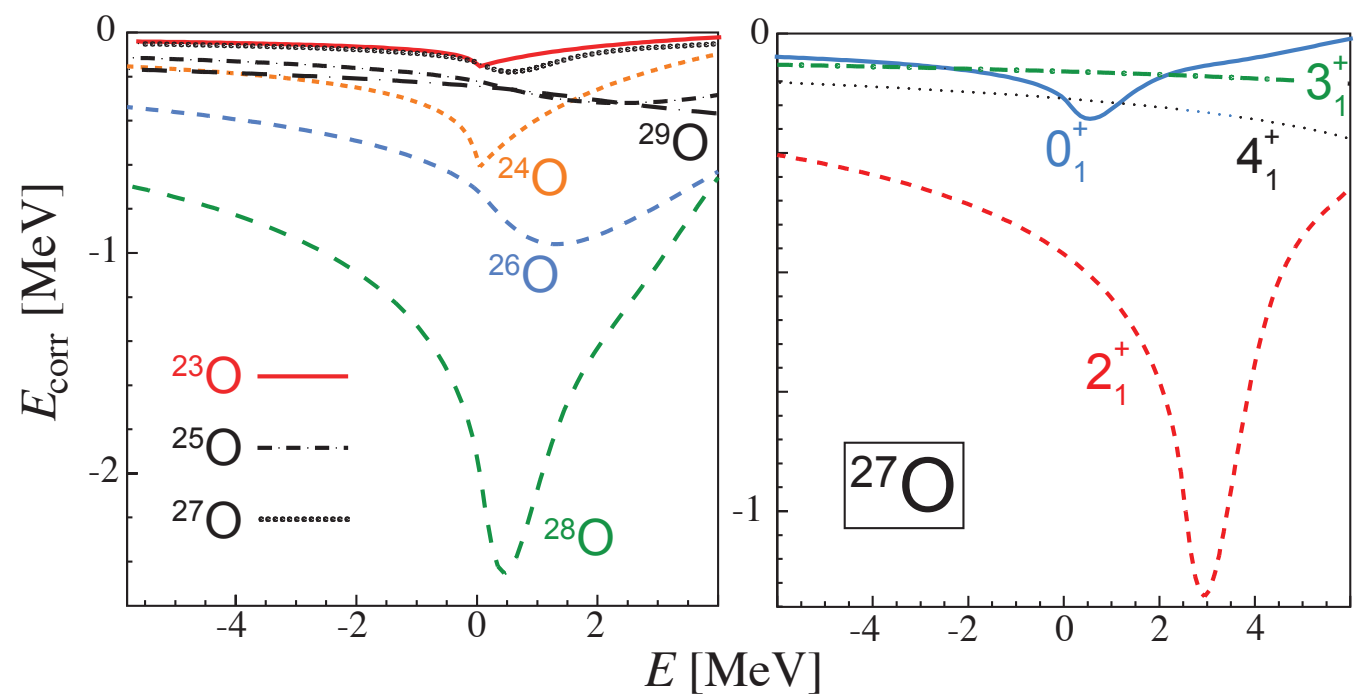

Figure 4. The continuum-coupling energy correction $E_{\text {corr }}(E)$. The value $E=0$ corresponds to the first (one-neutron emission) threshold. For $H_{Q Q}$, we take USD Hamiltonian for the $s d$ shell [53] and KB' interaction for the $p f$ shell [54]. The crossshell interaction is the G-matrix [55]. The coupling to continuum is given by the Wigner-Bartlett interaction with the strength $V_{0}=-414 \mathrm{MeV} \mathrm{fm}{ }^{3}$. Left: ground state configurations of ${ }^{23-29} \mathrm{O}$. Only a contribution from a single channel corresponding to a neutron coupled to the ground state in the daughter ${ }^{A-1} \mathrm{O}$ nucleus is included. Right: the ground state configuration of ${ }^{27} \mathrm{O}$. Contributions from couplings to various positive parity states in ${ }^{26} \mathrm{O}$ are indicated.

One can see that the continuum-coupling energy correction does depend on wave functions involved in the coupling matrix element, i.e., the wave function $\Psi_{i}$ in the parent nucleus $[N, Z]$ and the channel wave function $\left[{ }_{Z} X_{N-1}\left(I^{\pi}\right) \otimes{ }^{1} \mathrm{n}(l j)\right]_{E^{\prime}}^{J^{\pi}}$. The correction $E_{\text {corr }}$ varies strongly in a broad interval of energies around the neutron emission threshold: from $\pm 4 \mathrm{MeV}$ in ${ }^{28} \mathrm{O}$ to $\pm 2 \mathrm{MeV}$ in ${ }^{26} \mathrm{O}$ and ${ }^{24} \mathrm{O}$. As a result of the centrifugal barrier, a maximum of $\left|E_{\text {corr }}\right|$ is shifted above the threshold if the angular momentum $l$ of a neutron is different from zero. For $l=0$, the first derivative of $\left|E_{\text {corr }}(E)\right|$ is discontinuous at the neutron emission threshold. A similar shift can be caused by a Coulomb barrier if the continuum coupling involves charged particle emission channels. Another interesting observation is the odd-even staggering of $E_{\text {corr }}$ [40]: a blocking of the virtual scattering to the particle continuum by an odd nucleon diminishes the continuum correction to the binding energy in odd- $N$ nuclei.

Figure 4 (right) shows individual contributions to the continuum-coupling energy 
correction in ${ }^{27} \mathrm{O}$ coming from couplings to various reaction channels ${ }_{8} \mathrm{O}_{18}\left(I^{\pi}\right) \otimes$ $\left.{ }^{1} \mathrm{n}(l j)\right]_{E^{\prime}}^{J^{\pi}}$. The largest contribution to $E_{\text {corr }}$ comes from the coupling to the lowest physical inelastic channel: $\left.{ }_{8} \mathrm{O}_{18}\left(2_{1}^{+}\right) \otimes{ }^{1} \mathrm{n}(l j)\right]^{3 / 2^{+}}$. The same wave appears in the elastic channel $\left.{ }_{8} \mathrm{O}_{19}\left(3 / 2_{1}^{+}\right) \otimes{ }^{1} \mathrm{n}(l j)\right]^{0^{+}}$contribution to $E_{\text {corr }}$ in the neighboring nucleus ${ }^{28} \mathrm{O}$ (see Fig. 4 on the left).

The angular momentum dependence and charge dependence of the continuumcoupling correction around the particle-emission threshold can be explained in the same way as a characteristic threshold behavior of scattering and reaction cross sections [57] (Wigner cusp). Due to the unitarity of the $S$-matrix and the resulting flux conservation, Wigner's threshold effect has a broad impact on various channel wave functions due to channel coupling [58, 59, 60, 61, 62]. Hence, the branch point singularity at the opening of a given reaction channel can induce non-local correlations between wave functions in distant channels. This effect has been found experimentally, for example, in the coupling of the analogous channels in $(d, p)$ and $(d, n)$ reactions [63]. These channel-channel correlations are yet another manifestation of the eigenfunction correlations induced by the presence of branch points or exceptional points.

Recently, it has been found that a threshold law, similar to the Wigner's law for scattering and reaction cross-sections, holds for one-nucleon overlap amplitudes [64], which probe shell occupancies; hence, they characterize many-body correlations. An immediate consequence of this finding is that the influence of the branch point on the configuration mixing in weakly bound mirror states will be different, even for identical separation energies in mirror states [65].

The size of the energy correction is a measure of the mixing of unperturbed ISM wave functions due to the coupling to the reaction channel(s), either open or closed. For ISM wave functions below all reaction thresholds, only the Hermitian part of the coupling term $W(E)$ acts. For unbound ISM wave functions, the competition of Hermitian $W^{R}$ and anti-Hermitian $W^{I}$ parts is an essential ingredient of the formation mechanism of exceptional points in the spectra of resonances. As shown above, $\left|E_{\text {corr }}\right|$ is both stateand system-dependent, i.e., the continuum coupling does not induce a global instability of all ISM eigenstates close to the particle emission threshold. On the contrary, for those ISM wave functions which are susceptible to the continuum coupling, the near-threshold instability is seen predominantly in a single pair of unperturbed ISM states. Other ISM states with the same quantum numbers remain spectators even though all of them are interconnected by the coupling to the same reaction channel(s). Both avoided level crossings (or exceptional points) and branch points associated with reaction thresholds induce the configuration mixing of continuum states. For weakly bound states, only branch point(s) can contribute to the configuration mixing.

In general, the mixing mechanism associated with the coupling of ISM eigenstates to the reaction channel(s) enhances the similarity of a bound, near-threshold ISM state $\Psi\left(J^{\pi}\right)$ with the decay channel $\left[\Psi^{(d)}\left(I^{\pi}\right) \otimes\left(\tau_{z} l j\right)\right]^{J^{\pi}}$, i.e., increases the corresponding spectroscopic factor $\left\langle\tilde{\Psi}\left(J^{\pi}\right) \| a_{l j}^{+}|| \Psi^{(d)}\left(I^{\pi}\right)\right\rangle^{2}$, where $\Psi^{(d)}$ is the wave function of the daughter system. (The tilde symbol above bra vector signifies that the complex 
conjugation in the dual space affects the angular part and leaves the radial part unchanged.) This 'alignment' of a near-threshold eigenstate of the effective Hamiltonian with the corresponding reaction channel has been demonstrated in the approximation of a one-nucleon continuum [56]. The phenomenon is based on three general principles: (i) the branch point nature of the reaction channel threshold, (ii) the unitarity of the $S$-matrix, and (iii) the $2 \times 2$ matrix structure in the eigenfunction mixing. These principles neither invoke any particular interaction in the internal space nor any special structure of eigenfunctions involved in the mutual coupling. Hence, the alignment of near-threshold eigenfunctions of the effective Hamiltonian with the reaction channel should be present in any open quantum many-body system which has complex reaction channels. In particular, the entanglement of ISM wave functions due to their coupling to a common particle emission channel should be responsible for the omnipresence of clustering phenomena in states close to their cluster decay thresholds. Indeed, as illustrated in Fig. 5, $\alpha$-cluster states have been found among the ISM wave functions close to the $\alpha$-particle emission thresholds in, for example, ${ }^{12} \mathrm{C}$, whereas correlated twoneutron pair has been seen in the halo ground state of ${ }^{11} \mathrm{Li}$ close to the two-neutron emission threshold.

$\alpha$ cluster states

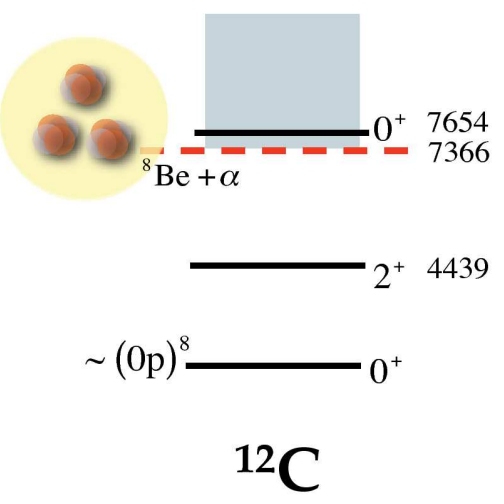

halo states

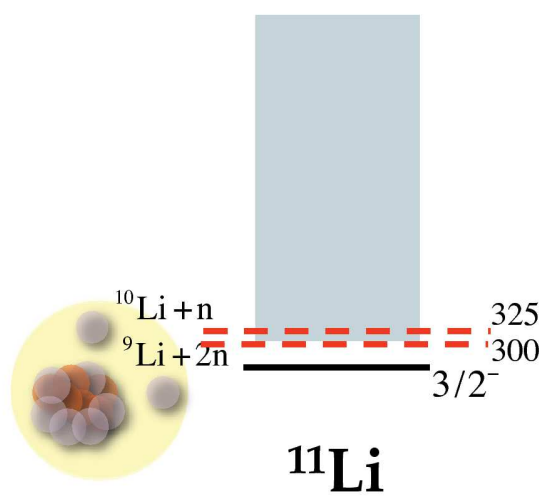

Figure 5. Cluster states close to decay thresholds. Left: Excited $0^{+}$state in ${ }^{12} \mathrm{C}$ (Hoyle state) just above the ${ }^{8} \mathrm{Be}+\alpha$ threshold. Right: Subthreshold halo ground state of ${ }^{11} \mathrm{Li}$.

The threshold features are generic: they are neither accidental nor arise from particular properties of the nuclear Hamiltonian. This universality forms a foundation of Ikeda systematics [66] which can be extended naturally to halo phenomena, see Fig. 5 . In this formulation, Borromean systems correspond to an example of clustering where the cluster itself is not a self-bound system.

We are still far away from a quantitative understanding of the organization of ISM eigenfunctions near the particle emission threshold. The structure of the ground state wave function of ${ }^{11} \mathrm{Li}$ is a particularly interesting case-study due to an intricate 
sequence of low-lying two- and one-neutron emission thresholds which are separated by less than $30 \mathrm{keV}($ !). This approximate degeneracy of two different emission thresholds may have important consequences on the structure of halo two-neutron pair. The relative weights of configurations, such as $\left[{ }^{9} \mathrm{Li}\left(3 / 2^{-}\right)\right] \otimes\left[\mathrm{n}^{2}\left(0^{+}\right)\right]$, corresponding to the two-neutron continuum of ${ }^{9} \mathrm{Li}$, and $\left\{\left[{ }^{9} \mathrm{Li}\left(1 / 2^{-}\right)\right] \otimes\left[\mathrm{n}\left(1 / 2^{-}\right)\right]\right\}^{1^{+}} \otimes\left[\mathrm{n}\left(1 / 2^{-}\right)\right]$, $\left\{\left[{ }^{9} \operatorname{Li}\left(1 / 2^{-}\right)\right] \otimes\left[\mathrm{n}\left(1 / 2^{+}\right)\right]\right\}^{1^{-}} \otimes\left[\mathrm{n}\left(1 / 2^{+}\right)\right]$, or $\left\{\left[{ }^{9} \operatorname{Li}\left(3 / 2^{-}\right)\right] \otimes\left[\mathrm{n}\left(1 / 2^{-}\right)\right]\right\}^{2^{+}} \otimes\left[\mathrm{n}\left(1 / 2^{-}\right)\right]$, associated with an unbound ${ }^{10} \mathrm{Li}$, reflects the influence of these two almost degenerate branch points on the structure of the ground state wave function of ${ }^{11} \mathrm{Li}$.

The exceptional points, whose dramatic effects on the phase rigidity can be seen in Fig. 3, are also generic in Hamiltonian systems. They arise as a result of an interplay between Hermitian and non-Hermitian parts of the continuum coupling term $W(E)$ for energies above the elastic channel threshold. The number of exceptional points depends only on some basic features of the system, such as its dimensionality and quantum integrability [67]. On the contrary, the precise position of any exceptional point in the spectrum of eigenvalues of the energy-dependent effective Hamiltonian depends on the choice of the ISM Hamiltonian $H_{Q Q}$, the strength of the coupling term $W(E)$, and the energy $E$ of the system. Hence, its finding provides the test of both the effective interaction and the configuration mixing in any OQS.

It has been found recently that low-energy exceptional points appear for realistic values of coupling to the continuum and hence could be accessible experimentally [52]. At low excitation energies, they could be seen, for example, as individual peaks associated with a jump by $2 \pi$ of the elastic scattering phase shift. Also in the neighborhood of an exceptional point for avoided crossing of resonances, this characteristic imprint of the scattering phase shift remains, which gives a real chance that traces of the exceptional could actually be searched for in reaction studies.

Complex and biorthogonal eigenstates of $\mathcal{H}_{Q Q}(E)$ provide a natural basis in which one can express the resonant part of any scattering wave function:

$$
\Upsilon_{E}^{c}=\zeta_{E}^{c}+\sum_{j} a_{j} \tilde{\Psi}_{j}
$$

where $a_{j}=\frac{\left\langle\Psi_{\tilde{j}}\left|H_{Q P}\right| \zeta_{E}^{c}\right\rangle}{\left(E-E_{j}\right)}$ and $\tilde{\Psi}_{j} \equiv\left(1+G_{P}^{(+)} H_{P Q}\right) \Psi_{j}$. Dominant contributions to $\Upsilon_{E}^{c}$ inside an interaction region are given by eigenfunctions $\Psi_{j}$ of $\mathcal{H}_{Q Q}$, i.e.,

$$
\Upsilon_{E}^{c} \sim \sum_{j} a_{j} \Psi_{j}
$$

Both branch points (reaction thresholds) and double poles of the $S$ matrix (exceptional points) lead to a non-separable entanglement of two eigenstates of the effective Hamiltonian which manifests itself in a singular behavior of matrix elements of any operator which does not commute with $\mathcal{H}_{Q Q}$ [52, 67, 68]. These unusual features are yet another facet of the profound change of the nature of ISM states close to the reaction thresholds and avoided resonance crossings which are responsible for clustering and strong mixing in OQSs. 


\subsection{Future developments: happy marriage of nuclear structure and nuclear reactions}

To understand the formation of a network of correlated states, e.g., the chain of isotopes from a well-bound ${ }^{9} \mathrm{Li}$ to an unbound ${ }^{12} \mathrm{Li}$, a structure of the Hoyle resonance, and the radiative capture reactions, such ${ }^{12} \mathrm{C}(\alpha, \gamma){ }^{16} \mathrm{O}$, a theoretical framework is required that would provide a unified approach to nuclear structure and reactions. Such a theoretical framework could be developed based on the GSM [23, 24, 25, 26]. This model, formulated in the Rigged Hilbert Space [28, 29] and using a complete Berggren ensemble [27], is a generalization of the nuclear ISM for a description of bound states, resonances, and many-body scattering continuum. In a nuclear structure application, solutions of the GSM can be found by diagonalizing a complex-symmetric Hamiltonian matrix. The 'dimensional catastrophe' in GSM when increasing the number of active particles is much more serious than in the standard ISM because each single-particle continuum state in the Berggren ensemble becomes a new shell in the many-body GSM formulation. This acute problem has been alleviated by recent progress in the generalization of the Density Matrix Renormalization Group [69, 70, 71] method to non-Hermitian, complexsymmetric matrix problems [72, 73].

Significant progress has also been made in applications of realistic interactions in GSM [74]. Finally, powerful techniques for a selection of physical resonances, based on the overlap method, have been developed [23, 25]. Still, much work is needed to develop appropriate effective interactions which would allow a systematic study of the structure of nuclear states in long isotopic or isotonic chains: from the valley of stability towards particle drip lines.

So far, most applications of the GSM have addressed nuclear structure phenomena. Further progress can only be achieved if the method could be fully extended to reaction problems. This can be achieved by the coupled-channel formulation of the scattering problem:

$$
\int d r^{\prime} \sum_{c^{\prime}} \sum_{m, n}\left\langle c ; r \mid \Psi_{n}\right\rangle\left[\left\langle\Psi_{\tilde{n}}\left|H-E \delta_{n m}\right| \Psi_{m}\right\rangle\right]\left\langle\Psi_{\tilde{m}} \mid c^{\prime} ; r^{\prime}\right\rangle \Omega_{c^{\prime}}\left(r^{\prime}\right)=0
$$

A similar approach, based on the Resonating Group Method [75, 76, 77], has been developed in the no-core shell-model approach to nuclear scattering problems [78, 79]. In the above equation, $H_{n m} \equiv\left\langle\Psi_{n}|H| \Psi_{m}\right\rangle$ is the GSM Hamiltonian matrix and $O_{c ; n} \equiv\left\langle c ; r \mid \Psi_{n}\right\rangle$ are overlaps of the reaction channel and the GSM many-body state. Formally, Eq. (8) takes the same form independently of the choice of reaction channels; the whole complication is hidden in the overlaps $O_{c ; n}$. Their calculation is a formidable task for the GSM approach. It requires a complicated change of the coordinate system which makes the calculation difficult for heavier projectiles, even for reactions with two-

body asymptotic conditions. Any future progress in the application of GSM to nuclear reactions is ultimately related to the progress in the development of new algorithms for fast calculation of overlaps. 
Open problems in theory of nuclear open quantum systems

\section{Acknowledgments}

We wish to thank J. Rotureau for useful discussions. This work was supported in part by the U.S. Department of Energy under Contract No. DE-FG02-96ER40963 (University of Tennessee) and by the CICYT-IN2P3 cooperation.

\section{References}

[1] Göppert-Mayer M 1949 Phys. Rev. 751969

[2] Haxel O, Jensen J.H.D. and Süss H.E. 1949 Phys. Rev. 751766

[3] Lane A.M. 1955 Proc. R. Soc. A 68197

[4] Kurath D 1956 Phys. Rev. 101216

[5] Caurier E, Martinez-Pinedo G, Nowacki F Poves A and Zuker A.P. 2005 Rev. Mod. Phys. 77427

[6] Wilkinson D.H. 1957 Proceedings of the Robert A. Welsh Foundation Conference on Chemical Research. I. The Structure of the Nucleus, Houston, TX 13

[7] Bohr A and Mottelson B 1975 Nuclear Structure, Vol. II (Benjamin, Reading, MA)

[8] Feshbach H 1958 Ann. Phys., NY 5357

[9] Feshbach H 1962 Ann. Phys., NY 19287

[10] Brenig W 1959 Nucl. Phys. 13333

[11] Rodberg L.S. 1961 Phys. Rev. 124210

[12] Macdonald W.M. 1964 Nucl. Phys. 54393 and $\mathbf{5 6} 636$

[13] Fano U 1961 Phys. Rev. 1241866

[14] Barrett R.F., Biedenharn L.C., Danos M, Delsanto P.P. and Greiner W 1973 Rev. Mod. Phys. 45 44

[15] Mahux C and Weidenmüller H.A. 1969 Shell Model Approach to Nuclear Reactions (North-Holland, Amsterdam)

[16] Barz H.W., Rotter I and Höhn J 1977 Nucl. Phys. A 275111

[17] Philpott R.J. 1977 Fizika 9109

[18] Rotter I, Barz H.W. and Höhn J 1978 Nucl. Phys. A 297237

[19] Bennaceur K, Nowacki F, Okołowicz J and Płoszajczak M 1999 Nucl. Phys. A 651289

[20] Volya A and Zelevinsky V 2006 Phys. Rev. C 74064314

[21] Rotureau J, Okołowicz J and Płoszajczak M 2006 Nucl. Phys. A 76713

[22] Okołowicz J, Płoszajczak M and Rotter I 2003 Physics Reports 374271

[23] Michel N, Nazarewicz W, Płoszajczak M and Bennaceur K 2002 Phys. Rev. Lett. 89042502

[24] Id Betan R, Liotta R.J., Sandulescu N and Vertse T 2002 Phys. Rev. Lett. 89042501

[25] Michel N, Nazarewicz W, Płoszajczak M and Okołowicz J 2003 Phys. Rev. C 67054311

[26] Michel N, Nazarewicz W and Płoszajczak M 2004 Phys. Rev. C 70064313

[27] Berggren T 1968 Nucl. Phys. A 109265

[28] Gel'fand I.M. and Vilenkin N.Ya. 1961 Generalized Functions, Vol. 4 (Academic Press, New York)

[29] Maurin K 1968 Generalized Eigenfunction Expansions and Unitary Representations of Topological Groups (Polish Scientific Publishers, Warsaw)

[30] Gamow G 1928 Z. Phys. 51204

[31] Gurney R.W. and Condon E.U. 1929 Phys. Rev. 33127

[32] Michel N, Nazarewicz W, Płoszajczak M and Vertse T 2009 J. Physique G 36013101

[33] Navrátil P and Barrett B.R. 1996 Phys. Rev. C 542986

[34] Navrátil P and Barrett B.R. 2000 Phys. Rev. C 62054311

[35] Roth R and Navrátil P 2007 Phys. Rev. Lett. 99092501

[36] Dobaczewski J, Michel N, Nazarewicz W, Płoszajczak M and Rotureau J 2007 Prog. Part. Nucl. Phys. 59432

[37] Stauffer D 1985 Introduction to Percolation Theory (Taylor \& Francis, London and Philadelphia) 
[38] Botet R and Płoszajczak M 2002 Universal Fluctuations - The Phenomenology of Hadronic Matter World Scientific Lecture Notes in Physics 65 (World Scientific, Singapore)

[39] Okołowicz J, Płoszajczak M and Luo Y 2007 Acta Physica Polonica B 39389

[40] Luo Y, Okołowicz and Płoszajczak M 2002 arXiv:nucl-th/0211068

[41] Otsuka T, Suzuki T, Holt J.D., Schwenk A and Akaishi Y 2009 arXiv:nucl-th/0908.2607

[42] Siegert A.F.J. 1939 Phys. Rev. 56750

[43] de la Madrid R 2005 Eur. J. Phys. 26287

[44] van Langen S.A., Brouwer P.W. and Beenakker C.W.J. 1997 Phys. Rev. E 55 R1

[45] Brouwer P.W. 2003 Phys. Rev. E 68046205

[46] Bulgakov E.N., Rotter I and Sadreev A.F. 2006 Phys. Rev. E 74056204

[47] Zuker A, Buck B and McGrory J.B. 1968 Phys. Rev. Lett. 2139

[48] Kato T 1995 Perturbation Theory for Linear Operators (Springer Verlag, Berlin)

[49] Zirnbauer M.R., Verbaarschot J.J.M. and Weidenmüller H.A. 1983 Nucl. Phys. A 411161

[50] Heiss W.D. and Steeb W.-H. 1991 J. Math. Phys. 323003

[51] Hernández E, Jáuregui A and Mondragón A 2003 Int. J. of Theor. Phys. 422167

[52] Okołowicz J and Płoszajczak M 2009 Phys. Rev. C 80034619

[53] Wildenthal B.H. 1984 Prog. Part. Nucl. Phys. 115

[54] Poves A and Zuker A 1981 Physics Reports 704

[55] Kahana S, Lee H.C. and Scott C.K. 1969 Phys. Rev. 180956

[56] Chatterjee R, Okołowicz J and Płoszajczak M 2006 Nucl. Phys. A 764528

[57] Wigner E.P. 1948 Phys. Rev. 731002

[58] Baz A.I. 1957 Sov. Phys. - JETP 6709

[59] Newton R.G. 1959 Phys. Rev. 1141611

[60] Meyerhof W.E. 1963 Phys. Rev. 129692

[61] Baz A.I., Zeldovich Y.B. and Perelomov A.M. 1969 Scattering, Reactions and Decays in Nonrelativistic Quantum Mechanics (Jerusalem)

[62] Hategan C 1978 Ann. Phys., NY 11677

[63] Moore C.F., Watson C.E., Zaidi S.A.A., Kent J.J. and Kulleck J.G. 1966 Phys. Rev. Lett. 17926

[64] Michel N, Nazarewicz W and Płoszajczak M 2007 Phys. Rev. C 75, 031301

[65] Michel N, Nazarewicz W and Płoszajczak M 2009 arXiv:0911.4902

[66] Ikeda K, Takigawa N and Horiuchi H 1968 Prog. Theor. Phys. Suppl. Extra Number

[67] Dukelsky J, Okołowicz J and Płoszajczak M 2009 J. Stat. Mech. L07001

[68] Okołowicz J and Płoszajczak M 2009 Acta Physics Polonica B 40409

[69] White S.R. 1992 Phys. Rev. Lett. 692363

[70] White S.R. 1993 Phys. Rev. B 4810345

[71] Carlon E, Henkel M and Schollwöck U 1991 Eur. J. Phys. B 1299

[72] Rotureau J, Michel N, Nazarewicz W, Płoszajczak M and Dukelsky J 2006 Phys. Rev. Lett. 97 110603

[73] Rotureau J, Michel N, Nazarewicz W, Płoszajczak M and Dukelsky 2009 Phys. Rev. C 79014304

[74] Hagen G, Hjorth-Jensen M and Michel N 2006 Phys. Rev. C 73064307

[75] Wildermuth K and Kanellopoulos Th 1958 Nucl. Phys. 7150

[76] Wildermuth K and Kanellopoulos Th 1959 Nucl. Phys. 9449

[77] Tang Y.C., LeMere M and Thompson D.R. 1978 Physics Reports 47167

[78] Quaglioni S and Navrátil P 2008 Phys. Rev. Lett. 101092501

[79] Quaglioni S and Navrátil P 2009 Phys. Rev. C 79044606 